\title{
On the convergence of a modified superquadratic method for generalized equations
}

\author{
Mohammed Harunor Rashid ${ }^{\mathrm{a}^{*}}$ and Md. Zulfiker Ali $^{\mathrm{b}}$ \\ ${ }^{\mathrm{a}, \mathrm{b}}$ Department of Mathematics, Faculty of Science, University of Rajshahi, Rajshahi-6205, Bangladesh \\ ${ }^{*}$ Corresponding authorE-mail: harun_math@ru.ac.bd
}

\section{Article Info \\ Keywords: Generalized equations, Lipschitz-like mappings, Semi-local convergence, Set-valued mappings. 2010 AMS: 47H04, 49J53, 65K10 \\ Received: 14 April 2018 \\ Accepted: 16 September 2018 \\ Available online: 30 September 2018}

\begin{abstract}
Let $X$ and $Y$ be Banach spaces. Let $\Omega$ be an open subset of $X$. Suppose that $f: X \rightarrow Y$ is Fréchet differentiable in $\Omega$ and $\mathscr{F}: X \rightrightarrows 2^{Y}$ is a set-valued mapping with closed graph. In the present paper, a modified superquadratic method (MSQM) is introduced for solving the generalized equations $0 \in f(x)+\mathscr{F}(x)$, and studied its convergence analysis under the assumption that the second Fréchet derivative of $f$ is Hölder continuous. Indeed, we show that the sequence, generated by MSQM, converges super-quadratically in both semi-locally and locally to the solution of the above generalized equation whenever the second Fréchet derivative of $f$ satisfies a Hölder-type condition.
\end{abstract}

\section{Introduction}

Throughout this paper we assume that $X$ and $Y$ are two real or complex Banach spaces and $\Omega \neq \emptyset$ is an open subset of $X$. Let $f: X \rightarrow Y$ be a Fréchet differentiable function on $\Omega$. Further, assume that the first and second Fréchet derivatives of $f$ are denoted by $\nabla f$ and $\nabla^{2} f$ respectively. Let $\mathscr{F}$ be a set-valued mapping with closed graph acting between Banach space $X$ and the subsets of $Y$. In this communication, we are interested to approximate the solution of the following generalized equation problem

$$
0 \in f(x)+\mathscr{F}(x)
$$

The inclusions type (1.1), introduced by Robinson [24,26] as a general tool for describing, analyzing, and solving different problems in a unified manner, have been studied extensively. The inclusion problem (1.1) is an abstract model for variety of problems. When $\mathscr{F}=\{0\}$, (1.1) is an equation. When $\mathscr{F}$ is the positive orthant in $\mathbb{R}^{n},(1.1)$ is a system of inequalities. When $\mathscr{F}$ is the normal cone to a convex and closed set in $X,(1.1)$ reduces to variational inequalities. When $\mathscr{F}=\partial \psi_{C}$ is the subdifferential of the function

$$
\psi_{C}(x)= \begin{cases}0, & \text { if } x \in C \\ +\infty, & \text { otherwise }\end{cases}
$$

(1.1) is reduced to some minimization problems which has been studied by Robinson [25].

To solve (1.1), Dontchev [1] introduced the following classical Newton-type method, for each $k=0,1, \ldots$,

$$
0 \in f\left(x_{k}\right)+\nabla f\left(x_{k}\right)\left(x_{k+1}-x_{k}\right)+\mathscr{F}\left(x_{k+1}\right),
$$

under the assumptions the set-valued mapping $\mathscr{F}$ is pseudo-Lipschitz and the Fréchet derivative of $f$ is Lipschitz on a neighborhood of the solution of (1.1) and established a quadratic convergence of the method. In his subsequent paper [2], he proved the uniform convergence of the method. By following Dontchev's method, Piétrus [5] obtained a super-linear convergence when the Fréchet derivative of $f$ is Hölder continuous on a neighborhood of the solution of (1.1) and later he [6] established the uniform convergence of this method in this mild differentiability context.

Let $x \in X$. By $\mathscr{D}(x)$, we symbolize the subset of $X$ which is defined by

$$
\mathscr{D}(x):=\left\{d \in X: 0 \in f(x)+\nabla f(x) d+\frac{1}{2} \nabla^{2} f(x) d^{2}+\mathscr{F}(x+d)\right\} .
$$


For finding an approximate solution of (1.1), the extension of Dontchev's indigenous work [3] was done by Geoffroy et al. [14]. Geoffroy and Pietrus [13] introduced the following superquadratic method (see Algorithm 1) for solving the generalized equation (1.1) and showed that it is locally superquadratic convergent:

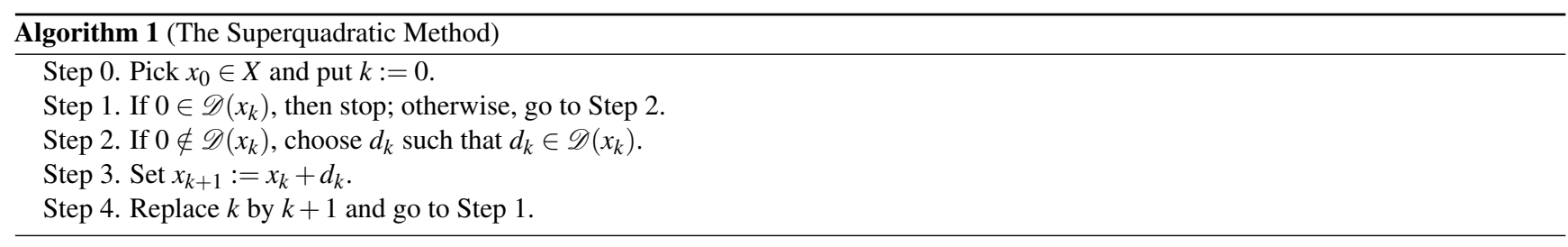

Note that under some suitable conditions around a solution $x^{*}$ of the generalized equation (1.1), the authors [13, Theorem 3.1] showed that there exists a neighborhood $\Omega$ of $x^{*}$ such that, for any point in $\Omega$, there exists a sequence generated by Algorithm 1 which is superquadratically convergent to the solution $x^{*}$. This implies that the convergence result, established in [13], guarantees the existence of a convergent sequence. Therefore, for any initial point near to a solution, the sequences generated by Algorithm 1 are not uniquely defined and not every generated sequence is convergent. Hence, in view of numerical computation, this kind of methods is not convenient in practical application. This drawback motivates us to propose a method 'so-called' modified superquadratic method (MSQM) as follows:

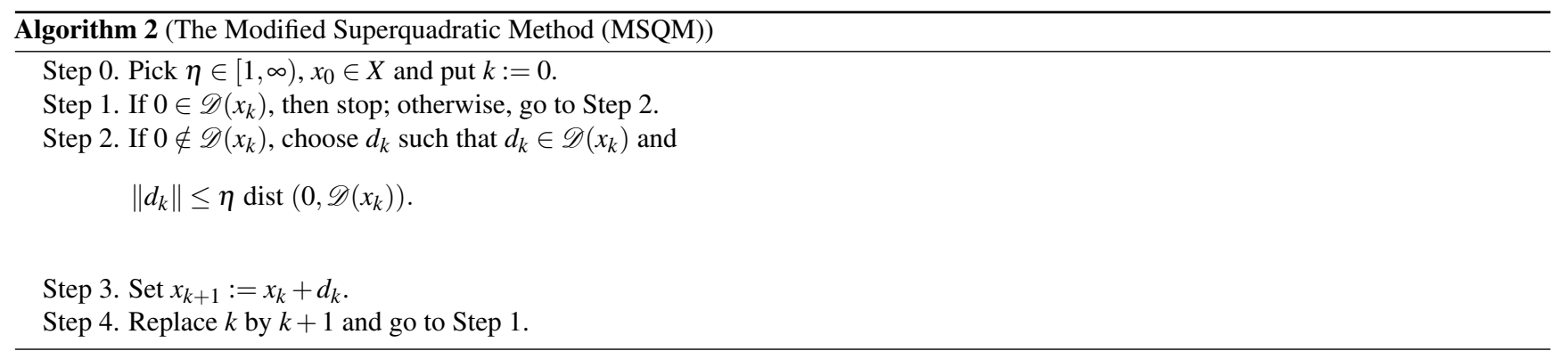

The difference between Algorithms 1 and 2 is that Algorithm 2 generates at least one sequence and every generated sequence is convergent but this does not appear in Algorithm 1. Since the sequences generated by Algorithm 1 are not uniquely defined, in contrast with Algorithm 1, we can guess that Algorithm 2 is more suitable than Algorithm 1 in numerical computation.

It is remark that if we replace the set $\mathscr{D}(x)$ by

$$
\mathscr{S}(x):=\{d \in X: 0 \in f(x)+\nabla f(x) d+\mathscr{F}(x+d)\},
$$

the Algorithm 2 introduced in the present paper will be the same with the Algorithm given in [16, 23].

To solving (1.1), there have a large number of works on semilocal analysis ; see for example [7, 8, 11, 12, 19, 20, 27, 28]. Rashid et al. [16, 23] established semilocal convergence analysis for solving the generalized equation problem (1.1), which was the extension of Dontchev's work in [1]. Rashid [17] introduced a variant of Newton-type Method for solving (1.1) and obtained its semilocal and local convergence results. The same author [18] associated extended Newton-type method for solving a variational inclusion of the form

$$
0 \in f(x)+g(x)+\mathscr{F}(x),
$$

where $g: X \rightarrow Y$ admits first order divided difference and established its semilocal and local convergence results for solving (1.1). As far as we know, there doesn't have any other study on semilocal analysis for the Algorithm 1.

The purpose of this study is to analyze the semilocal convergence for the modified superquadratic method defined by Algorithm 2 . The main tool is the Lipschitz-like property of set-valued mappings. The main results are the convergence criteria, established in Sect.3, which, based on the information around the initial point, provides some sufficient conditions ensuring the convergence to a solution of any sequence generated by Algorithm 2. As a consequence, local convergence result for the modified superquadratic method is obtained.

This paper is organized as follows: In Section 2, we recall a few necessary preliminary results. In Section 3, we consider the modified superquadratic method for solving the generalized equation as well as using the concept of Lipchitz-like mappings, we prove the existence of a sequence $\left\{x_{k}\right\}$ generated by Algorithm 2 and show that it is semilocally and locally superquadratic convergent. In the last section, we give a summary of the major results presented in this paper.

\section{Preliminary results}

Let $x \in X$ and $\mathbb{B}(x, r)=\{y:\|y-x\| \leq r\}$ be denote the closed ball centered at $x$ with radius $r>0$. Let $\Gamma: X \rightrightarrows 2^{Y}$ be a set-valued mapping. The domain of $\Gamma$, denoted by dom $\Gamma$, is defined by

$$
\operatorname{dom} \Gamma:=\{x \in X: \Gamma(x) \neq \emptyset\} .
$$

The inverse and the graph of $\Gamma$, denoted by $\Gamma^{-1}$ and gph $\Gamma$ respectively, are defined by

$$
\begin{aligned}
& \Gamma^{-1}(y):=\{x \in X: y \in \Gamma(x)\} \quad \text { for each } y \in Y \\
& \text { and } \operatorname{gph} \Gamma:=\{(x, y) \in X \times Y: y \in \Gamma(x)\} .
\end{aligned}
$$


Let $B \subseteq X$. The distance from a point $x \in X$ to a set $B$ is defined by

$$
\operatorname{dist}(x, B):=\inf _{b \in B}\|x-b\|,
$$

and the excess from the set $A$ to the set $B$ is defined by

$$
e(B, A)=\sup _{x \in B}\{\operatorname{dist}(x, A)\} .
$$

The notions of pseudo-Lipschitz and Lipchitz-like set-valued mappings are due to [23]. Aubin $[9,10]$ introduced these notions and studied extensively.

Definition 2.1. Let $G: Y \rightrightarrows 2^{X}$ be a set-valued mapping and let $(\bar{y}, \bar{x}) \in \operatorname{gph} G$. Let $r_{\bar{x}}>0, r_{\bar{y}}>0$ and $M>0$. Then the mapping $G$ is said to be

(a) Lipschitz-like on $\mathbb{B}\left(\bar{y}, r_{\bar{y}}\right)$ relative to $\mathbb{B}\left(\bar{x}, r_{\bar{x}}\right)$ with constant $M$ if the following inequality holds:

$$
e\left(G\left(y_{1}\right) \cap \mathbb{B}\left(\bar{x}, r_{\bar{x}}\right), G\left(y_{2}\right)\right) \leq M\left\|y_{1}-y_{2}\right\| \quad \text { for any } y_{1}, y_{2} \in \mathbb{B}\left(\bar{y}, r_{\bar{y}}\right) .
$$

(b) pseudo-Lipschitz around $(\bar{y}, \bar{x})$ if there exist constants $a>0, b>0$ and $M^{\prime}>0$ such that $G$ is Lipschitz-like on $\mathbb{B}(\bar{y}, b)$ relative to $\mathbb{B}(\bar{x}, a)$ with constant $M^{\prime}$.

The following notion of $(L, p)$-Hölder continuity property is due to [21].

Definition 2.2. Let $f: X \rightarrow Y$ be a Fréchet differentiable function on some neighborhood $U$ of $\bar{x}$ and let $\nabla^{2} f$ be the second Fréchet derivative of $f$ on $U$. Let $p \in[0,1]$ and $L>0$. Then $\nabla^{2} f$ is called $(L, p)$-Höder continuous on $U$ with constant $L$ if the following condition holds:

$$
\left\|\nabla^{2} f\left(x_{1}\right)-\nabla^{2} f\left(x_{2}\right)\right\| \leq L\left\|x_{1}-x_{2}\right\|^{p} \text {, for any } x_{1}, x_{2} \in U \text {. }
$$

The following lemma has taken from [23]. This lemma employs a vital role for proving the convergence analysis.

Lemma 2.3. Let $G: Y \rightrightarrows 2^{X}$ be a set-valued mapping and let $(\bar{y}, \bar{x}) \in \operatorname{gph} G$. Assume that $G$ is Lipschitz-like on $\mathbb{B}\left(\bar{y}, r_{\bar{y}}\right)$ relative to $\mathbb{B}\left(\bar{x}, r_{\bar{x}}\right)$ with constant $M$. Then

$$
\operatorname{dist}(x, G(y)) \leq M \operatorname{dist}\left(y, G^{-1}(x)\right)
$$

holds for every $x \in \mathbb{B}\left(\bar{x}, r_{\bar{x}}\right)$ and $y \in \mathbb{B}\left(\bar{y}, \frac{r_{\bar{y}}}{3}\right)$ satisfying $\operatorname{dist}\left(y, G^{-1}(x)\right) \leq \frac{r_{\bar{y}}}{3}$.

We would like to finish this section with the following lemma that is known in [4].

Lemma 2.4. Let $\Phi: X \rightrightarrows 2^{X}$ be a set-valued mapping. Let $\bar{x} \in X, c>0$ and $0<r<1$ be such that

$$
\operatorname{dist}(\bar{x}, \Phi(\bar{x}))<c(1-r) \text {; }
$$

and

$$
e\left(\Phi\left(x_{1}\right) \cap \mathbb{B}(\bar{x}, c), \Phi\left(x_{2}\right)\right) \leq r\left\|x_{1}-x_{2}\right\| \quad \text { for any } x_{1}, x_{2} \in \mathbb{B}(\bar{x}, c) .
$$

Then $\Phi$ has a fixed point in $\mathbb{B}(\bar{x}, c)$, that is, there exists $x \in \mathbb{B}(\bar{x}, c)$ such that $x \in \Phi(x)$. Moreover, if $\Phi$ is single-valued, then the fixed point of $\Phi$ in $\mathbb{B}(\bar{x}, c)$ is unique.

\section{Convergence analysis of MSQM}

This section is devoted to prove the existence and convergence of the sequences generated by the modified superquadratic method defined by Algorithm 2. To this end, let $x \in X$ and let us define the mapping $T_{x}$ by

$$
T_{x}(\cdot):=f(x)+\nabla f(x)(\cdot-x)+\frac{1}{2} \nabla^{2} f(x)(\cdot-x)^{2}+\mathscr{F}(\cdot) .
$$

Then for the construction of $\mathscr{D}(x)$, we have that

$$
\begin{aligned}
\mathscr{D}(x) & =\left\{d \in X: 0 \in T_{x}(x+d)\right\} \\
& =\left\{d \in X: x+d \in T_{x}^{-1}(0)\right\} .
\end{aligned}
$$

Moreover, for any $v \in X$ and $y \in Y$, the inclusions

$$
v \in T_{x}^{-1}(y) \text { and } y \in f(x)+\nabla f(x)(v-x)+\frac{1}{2} \nabla^{2} f(x)(v-x)^{2}+\mathscr{F}(v) .
$$

are equivalent. In particular,

$$
\bar{x} \in T_{\bar{x}}^{-1}(\bar{y}) \quad \text { for each }(\bar{x}, \bar{y}) \in \operatorname{gph}(f+\mathscr{F}) .
$$

The following result is due to [15]. This result establishes the equivalence relation between $(f+\mathscr{F})^{-1}$ and $T_{\bar{x}}^{-1}$.

Lemma 3.1. Let $f: X \rightarrow Y$ be a function and let $(\bar{x}, \bar{y}) \in \operatorname{gph}(f+\mathscr{F})$. Assume that $f$ is twice differentiable in an open neighborhood $\Omega$ of $\bar{x}$ and that its second-order derivative is continuous at $\bar{x}$. Then the following are equivalent: 
(i) The mapping $(f+\mathscr{F})^{-1}$ is pseudo-Lipschitz at $(\bar{y}, \bar{x})$;

(ii) The mapping $T_{\bar{x}}^{-1}(\cdot)$ is pseudo-Lipschitz at $(\bar{y}, \bar{x})$.

Let $r_{\bar{x}}>0, r_{\bar{y}}>0$ and $(\bar{x}, \bar{y}) \in \operatorname{gph}(f+\mathscr{F})$. Then, the closed graph property of the set-valued mapping $f+\mathscr{F}$ implies that $f+\mathscr{F}$ is continuous at $\bar{x}$ for $\bar{y}$, that is,

$$
\lim _{x \rightarrow \bar{x}} \operatorname{dist}(\bar{y}, f(x)+\mathscr{F}(x))=0 .
$$

Assume that $\mathbb{B}\left(\bar{x}, r_{\bar{x}}\right) \subseteq \Omega \cap \operatorname{dom} \mathscr{F}$. Moreover, by Lemma 3.1 we assume that the mapping $T_{\bar{x}}^{-1}$ is Lipschitz-like on $\mathbb{B}\left(\bar{y}, r_{\bar{y}}\right)$ relative to $\mathbb{B}\left(\bar{x}, r_{\bar{x}}\right)$ with constant $M$, that is,

$$
e\left(T_{\bar{x}}^{-1}\left(y_{1}\right) \cap \mathbb{B}\left(\bar{x}, r_{\bar{x}}\right), T_{\bar{x}}^{-1}\left(y_{2}\right)\right) \leq M\left\|y_{1}-y_{2}\right\| \forall y_{1}, y_{2} \in \mathbb{B}\left(\bar{y}, r_{\bar{y}}\right) .
$$

Let $p \in(0,1], L>0$ and setting

$$
\alpha:=\min \left\{r_{\bar{y}}-\frac{L\left(3^{p+2}+2^{p+2}\right) r_{\bar{x}}^{p+2}}{(p+1)(p+2) 2^{p+2}}, \frac{r_{\bar{x}}\left(2^{p+1}-5 M L r_{\bar{x}}^{p}\right)}{5 M 2^{p+2}}\right\} .
$$

Then

$$
\alpha>0 \text { if and only if } L<\min \left\{\frac{2^{p+2}(p+1)(p+2) r_{\bar{y}}}{\left(3^{p+2}+2^{p+2}\right) r_{\bar{x}}^{p+2}}, \frac{2^{p+1}}{5 M r_{\bar{x}}^{p}}\right\} .
$$

The following lemma plays a vital role for convergence analysis of the modified superquadratic method. The proof is a refinement of the one for [23, Lemma 3.1].

Lemma 3.2. Let $T_{\bar{x}}^{-1}$ be a Lipschitz-like mapping on $\mathbb{B}\left(\bar{y}, r_{\bar{y}}\right)$ relative to $\mathbb{B}\left(\bar{x}, r_{\bar{x}}\right)$ with constant $M$. Let $p \in(0,1]$ and $x \in \mathbb{B}\left(\bar{x}, \frac{r_{\bar{x}}}{2}\right)$. Assume that $\nabla f$ and $\nabla^{2} f$ are $(L, p)$-Höder continuous at $\bar{x}$ on $\mathbb{B}\left(\bar{x}, \frac{r_{\bar{x}}}{2}\right)$ with the same constant L defined by (3.6). Let $\alpha$ be defined in (3.5) so that (3.6) is satisfied. Then the mapping $T_{x}^{-1}$ is Lipschitz-like on $\mathbb{B}(\bar{y}, \alpha)$ relative to $\mathbb{B}\left(\bar{x}, \frac{r_{\bar{x}}}{2}\right)$ with constant $\frac{5 M 2^{p}}{2^{p+1}-5 M L r_{\bar{x}}^{p}}$ i.e.

$$
e\left(T_{x}^{-1}\left(t_{1}\right) \cap \mathbb{B}\left(\bar{x}, \frac{r_{\bar{x}}}{2}\right), T_{x}^{-1}\left(t_{2}\right)\right) \leq \frac{5 M 2^{p}}{2^{p+1}-5 M L r_{\bar{x}}^{p}}\left\|t_{1}-t_{2}\right\| \quad \text { for every } t_{1}, t_{2} \in \mathbb{B}(\bar{y}, \alpha) .
$$

Proof. Since $\alpha$ is defined in (3.5) so that (3.6) is satisfied, then it is clear that $\alpha>0$. Now let

$$
t_{1}, t_{2} \in \mathbb{B}(\bar{y}, \alpha) \quad \text { and } \quad u^{\prime} \in T_{x}^{-1}\left(t_{1}\right) \cap \mathbb{B}\left(\bar{x}, \frac{r_{\bar{x}}}{2}\right) .
$$

To complete the proof, it is sufficient to show that there exists $u^{\prime \prime} \in T_{x}^{-1}\left(t_{2}\right)$ such that

$$
\left\|u^{\prime}-u^{\prime \prime}\right\| \leq \frac{5 M 2^{p}}{2^{p+1}-5 M L r_{\bar{x}}^{p}}\left\|t_{1}-t_{2}\right\| .
$$

To finish this, we need to verify that there exists a sequence $\left\{x_{k}\right\} \subseteq \mathbb{B}\left(\bar{x}, r_{\bar{x}}\right)$ such that

$$
\begin{aligned}
t_{2} & \in f(x)+\nabla f(x)\left(x_{k-1}-x\right)+\nabla f(\bar{x})\left(x_{k}-x_{k-1}\right) \\
& +\frac{1}{2} \nabla^{2} f(x)\left(x_{k-1}-x\right)^{2}+\frac{1}{2} \nabla^{2} f(\bar{x})\left(\left(x_{k}-\bar{x}\right)^{2}-\right. \\
& \left.\left(x_{k-1}-\bar{x}\right)^{2}\right)+\mathscr{F}\left(x_{k}\right)
\end{aligned}
$$

and

$$
\left\|x_{k}-x_{k-1}\right\| \leq \frac{5 M}{2}\left\|t_{1}-t_{2}\right\|\left(\frac{5 M L r_{\bar{x}}^{p}}{2^{p+1}}\right)^{k-2}
$$

hold for each $k=2,3,4, \ldots$. We proceed by induction on $k$. Write

$$
\begin{aligned}
& a_{i}:=t_{i}-f(x)-\nabla f(x)\left(u^{\prime}-x\right)-\frac{1}{2} \nabla^{2} f(x)\left(u^{\prime}-x\right)^{2}+f(\bar{x}) \\
& +\nabla f(\bar{x})\left(u^{\prime}-\bar{x}\right)+\frac{1}{2} \nabla^{2} f(\bar{x})\left(u^{\prime}-\bar{x}\right)^{2} \quad \text { for each } i=1,2 .
\end{aligned}
$$

Note by (3.7) that

$$
\left\|x-u^{\prime}\right\| \leq\|x-\bar{x}\|+\left\|\bar{x}-u^{\prime}\right\| \leq r_{\bar{x}} .
$$

Furthermore, we have, for (3.10), that

$$
\begin{aligned}
\left\|a_{i}-\bar{y}\right\| & \leq\left\|t_{i}-\bar{y}\right\|+\| f\left(u^{\prime}\right)-f(x)-\nabla f(x)\left(u^{\prime}-x\right) \\
& -\frac{1}{2} \nabla^{2} f(x)\left(u^{\prime}-x\right)^{2}\|+\| f\left(u^{\prime}\right)-f(\bar{x}) \\
& -\nabla f(\bar{x})\left(u^{\prime}-\bar{x}\right)-\frac{1}{2} \nabla^{2} f(\bar{x})\left(u^{\prime}-\bar{x}\right)^{2} \| .
\end{aligned}
$$


If $\nabla f$ is $(L, p)$-Hölder continuous at $\bar{x}$ with constant $L$, then we have that

$$
\begin{aligned}
\|f(x)-f(\bar{x})-\nabla f(\bar{x})(x-\bar{x})\| & =\left\|\int_{0}^{1}[\nabla f(\bar{x}+t(x-\bar{x}))-\nabla f(\bar{x})](x-\bar{x}) d t\right\| \\
& \leq \int_{0}^{1}\|\nabla f(\bar{x}+t(x-\bar{x}))-\nabla f(\bar{x})\|\|x-\bar{x}\| d t \\
& \leq L\|x-\bar{x}\|^{p+1} \int_{0}^{1} t^{p} d t \\
& =\frac{L}{p+1}\|x-\bar{x}\|^{p+1} .
\end{aligned}
$$

Analogously, if $\nabla^{2} f$ is $(L, p)$-Hölder continuous at $\bar{x}$ with constant $L$, then we have that

$$
\begin{aligned}
\left\|f(x)-f(\bar{x})-\nabla f(\bar{x})(x-\bar{x})-\frac{1}{2} \nabla^{2} f(\bar{x})(x-\bar{x})^{2}\right\| & =\left\|\int_{0}^{1}\left[\nabla f(\bar{x}+t(x-\bar{x}))-\nabla f(\bar{x})-\nabla^{2} f(\bar{x})(\bar{x}+t(x-\bar{x})-\bar{x})\right](x-\bar{x}) d t\right\| \\
& \leq \int_{0}^{1}\left\|\nabla f(\bar{x}+t(x-\bar{x}))-\nabla f(\bar{x})-\nabla^{2} f(\bar{x})(\bar{x}+t(x-\bar{x})-\bar{x})\right\|\|x-\bar{x}\| d t \\
& =\int_{\bar{x}}^{x}\left\|\nabla f(u)-\nabla f(\bar{x})-\nabla^{2} f(\bar{x})(u-\bar{x})\right\| d u \\
& =\int_{\bar{x}}^{x} \int_{0}^{1}\left\{\left\|\nabla^{2} f(\bar{x}+s(u-\bar{x}))-\nabla^{2} f(\bar{x})\right\| d s\right\}\|u-\bar{x}\| d u \\
& \leq \int_{\bar{x}}^{x}\left\{L\|u-\bar{x}\|^{p+1} \int_{0}^{1} s^{p} d s\right\} d u \\
& \leq \frac{L}{p+1} \int_{\bar{x}}^{x}\|u-\bar{x}\|^{p+1} d u \\
& =\frac{L}{(p+1)(p+2)}\|x-\bar{x}\|^{p+2} .
\end{aligned}
$$

Then from (3.12), using the relations in (3.7), (3.11) and the relation $\alpha \leq r_{\bar{y}}-\frac{L\left(3^{p+2}+2^{p+2}\right) r_{\bar{x}}^{p+2}}{(p+1)(p+2) 2^{p+2}}$ by (3.5), we have that

$$
\begin{aligned}
\left\|a_{i}-\bar{y}\right\| & \leq \alpha+\frac{L}{(p+1)(p+2)}\left(\left\|u^{\prime}-x\right\|^{p+2}+\left\|u^{\prime}-\bar{x}\right\|^{p+2}\right) \\
& \leq \alpha+\frac{L}{(p+1)(p+2)}\left(r_{\bar{x}}^{p+2}+\frac{r_{\bar{x}}^{p+2}}{2^{p+2}}\right) \\
& =\alpha+\frac{L\left(1+2^{p+2}\right) r_{\bar{x}}^{p+2}}{(p+1)(p+2) 2^{p+2}} \leq r_{\bar{y}} .
\end{aligned}
$$

That is $a_{i} \in \mathbb{B}\left(\bar{y}, r_{\bar{y}}\right)$ for each $i=1,2$. Define $x_{1}:=u^{\prime}$. Then $x_{1} \in T_{x}^{-1}\left(t_{1}\right)$ by (3.7) and it follows from (3.2) that

$$
t_{1} \in f(x)+\nabla f(x)\left(x_{1}-x\right)+\frac{1}{2} \nabla^{2} f(x)\left(x_{1}-x\right)^{2}+\mathscr{F}\left(x_{1}\right)
$$

This can be written in another form as follows:

$$
\begin{aligned}
& \left.t_{1}+f(\bar{x})+\nabla f(\bar{x})\left(x_{1}-\bar{x}\right)\right)+\frac{1}{2} \nabla^{2} f(\bar{x})\left(x_{1}-\bar{x}\right)^{2} \in f(x) \\
& +\nabla f(x)\left(x_{1}-x\right)+\frac{1}{2} \nabla^{2} f(x)\left(x_{1}-x\right)^{2}+\mathscr{F}\left(x_{1}\right)+f(\bar{x}) \\
& +\nabla f(\bar{x})\left(x_{1}-\bar{x}\right)+\frac{1}{2} \nabla^{2} f(\bar{x})\left(x_{1}-\bar{x}\right)^{2} .
\end{aligned}
$$

This, by the definition of $a_{1}$, implies that

$$
a_{1} \in f(\bar{x})+\nabla f(\bar{x})\left(x_{1}-\bar{x}\right)++\frac{1}{2} \nabla^{2} f(\bar{x})\left(x_{1}-\bar{x}\right)^{2}+\mathscr{F}\left(x_{1}\right) .
$$

Hence $x_{1} \in T_{\bar{x}}^{-1}\left(a_{1}\right)$ by (3.2). This together with (3.7) implies that

$$
x_{1} \in T_{\bar{x}}^{-1}\left(a_{1}\right) \cap \mathbb{B}\left(\bar{x}, r_{\bar{x}}\right) .
$$

Noting that $a_{1}, a_{2} \in \mathbb{B}\left(\bar{y}, r_{\bar{y}}\right)$ and $T_{\bar{x}}^{-1}$ is Lipschitz-like by our assumption. Then it follows from (3.4) that there exists $x_{2} \in T_{\bar{x}}^{-1}\left(a_{2}\right)$ such that

$$
\left\|x_{2}-x_{1}\right\| \leq M\left\|a_{1}-a_{2}\right\|=M\left\|t_{1}-t_{2}\right\|<\frac{5 M}{2}\left\|t_{1}-t_{2}\right\| .
$$

Moreover, by the construction of $t_{2}$ and noting $x_{1}=u^{\prime}$, we have

$$
x_{2} \in T_{\bar{x}}^{-1}\left(a_{2}\right)=T_{\bar{x}}^{-1}\left(t_{2}-f(x)-\nabla f(x)\left(x_{1}-x\right)-\frac{1}{2} \nabla^{2} f(x)\left(x_{1}-x\right)^{2}+f(\bar{x})+\nabla f(\bar{x})\left(x_{1}-\bar{x}\right)+\frac{1}{2} \nabla^{2} f(\bar{x})\left(x_{1}-\bar{x}\right)^{2}\right),
$$


which, together with (3.2), implies that

$$
t_{2} \in f(x)+\nabla f(x)\left(x_{1}-x\right)+\nabla f(\bar{x})\left(x_{2}-x_{1}\right)+\frac{1}{2} \nabla^{2} f(x)\left(x_{1}-x\right)^{2}+\frac{1}{2} \nabla^{2} f(\bar{x})\left(\left(x_{2}-\bar{x}\right)^{2}-\left(x_{1}-\bar{x}\right)^{2}\right)+\mathscr{F}\left(x_{2}\right) .
$$

This shows that (3.8) and (3.9) are hold with generated points $x_{1}, x_{2}$.

Assume that $x_{1}, x_{2}, \ldots, x_{n}$ are obtained so that (3.8) and (3.9) are hold for $k=2,3, \ldots, n$. We need to construct $x_{n+1}$ such that (3.8) and (3.9) are also true for $k=n+1$. For this purpose, set

$$
\begin{aligned}
a_{i}^{n} & :=t_{2}-f(x)-\nabla f(x)\left(x_{n+i-1}-x\right)-\frac{1}{2} \nabla^{2} f(x)\left(x_{n+i-1}-x\right)^{2}+f(\bar{x}) \\
& +\nabla f(\bar{x})\left(x_{n+i-1}-\bar{x}\right)+\frac{1}{2} \nabla^{2} f(\bar{x})\left(x_{n+i-1}-\bar{x}\right)^{2} \quad \text { for each } i=0,1 .
\end{aligned}
$$

Then, for $i=0,1$, we obtain that

$$
\begin{aligned}
\left\|a_{0}^{n}-a_{1}^{n}\right\| & =\|(\nabla f(x)-\nabla f(\bar{x}))\left(x_{n}-x_{n-1}\right)+\frac{1}{2} \nabla^{2} f(x)\left(\left(x_{n}-x\right)^{2}-\left(x_{n-1}-x\right)^{2}\right) \\
& -\frac{1}{2} \nabla^{2} f(\bar{x})\left(\left(x_{n}-\bar{x}\right)^{2}-\left(x_{n-1}-\bar{x}\right)^{2}\right) \| \\
& =\|(\nabla f(x)-\nabla f(\bar{x}))\left(x_{n}-x_{n-1}\right)+\frac{1}{2} \nabla^{2} f(x)\left(\left(x_{n}-x_{n-1}+x_{n-1}-x\right)^{2}\right. \\
& \left.-\left(x_{n-1}-x\right)^{2}\right)-\frac{1}{2} \nabla^{2} f(\bar{x})\left(\left(x_{n}-x_{n-1}+x_{n-1}-\bar{x}\right)^{2}-\left(x_{n-1}-\bar{x}\right)^{2}\right) \| \\
& \leq\|\nabla f(x)-\nabla f(\bar{x})\|\left\|x_{n}-x_{n-1}\right\|+\frac{1}{2}\left\|\nabla^{2} f(x)-\nabla^{2} f(\bar{x})\right\|\left\|x_{n}-x_{n-1}\right\|^{2} \\
& +\left\|\nabla^{2} f(x)\left(x_{n-1}-x\right)-\nabla^{2} f(\bar{x})\left(x_{n-1}-\bar{x}\right)\right\|\left\|x_{n}-x_{n-1}\right\| .
\end{aligned}
$$

For all $z \in \mathbb{B}\left(\bar{x}, \frac{r_{\bar{x}}}{2}\right), x \mapsto \nabla f(x), x \mapsto \nabla^{2} f(x)$ and $x \mapsto \nabla^{2} f(x)(z-x)$ are $(L, p)$-Hölder continuous at $\bar{x}$, thus we have that

$$
\begin{aligned}
\left\|a_{0}^{n}-a_{1}^{n}\right\| & \leq L\|x-\bar{x}\|^{p}\left\{\left\|x_{n}-x_{n-1}\right\|+\frac{1}{2}\left\|x_{n}-x_{n-1}\right\|^{2}+\left\|x_{n}-x_{n-1}\right\|\right\} \\
& \leq \frac{L r_{\bar{x}}^{p}}{2^{p}}\left(2\left\|x_{n}-x_{n-1}\right\|+\frac{1}{2}\left\|x_{n}-x_{n-1}\right\|^{2}\right) \\
& \leq \frac{\operatorname{Lr} r_{\bar{x}}^{p}}{2^{p}}\left(2\left\|x_{n}-x_{n-1}\right\|+\frac{1}{2}\left\|x_{n}-x_{n-1}\right\|\right), \text { if the ball } \\
& \mathbb{B}\left(\bar{x}, \frac{r_{\bar{x}}}{2}\right) \text { is sufficiently small } \\
& =\frac{5 L r_{\bar{x}}^{p}}{2^{p+1}}\left\|x_{n}-x_{n-1}\right\| .
\end{aligned}
$$

Since $\left\|x_{1}-\bar{x}\right\| \leq \frac{r_{\bar{x}}}{2}$ by (3.7) and $\left\|t_{1}-t_{2}\right\| \leq 2 \alpha$ by (3.7), it follows from (3.9) that

$$
\begin{aligned}
\left\|x_{n}-\bar{x}\right\| & \leq \sum_{j=2}^{n}\left\|x_{j}-x_{j-1}\right\|+\left\|x_{1}-\bar{x}\right\| \\
& \leq 5 M \alpha \sum_{j=2}^{n}\left(\frac{5 M L r_{\bar{x}}^{p}}{2^{p+1}}\right)^{j-2}+\frac{r_{\bar{x}}}{2} \\
& =\frac{5 M \alpha 2^{p+1}}{2^{p+1}-5 M L r_{\bar{x}}^{p}}+\frac{r_{\bar{x}}}{2} .
\end{aligned}
$$

By (3.5), we have $\alpha \leq \frac{r_{\bar{x}}\left(2^{p+1}-5 M L r_{\bar{x}}^{p}\right)}{5 M 2^{p+2}}$ and so

$$
\left\|x_{n}-\bar{x}\right\| \leq r_{\bar{x}}
$$

Therefore, we obtain that

$$
\left\|x_{n}-x\right\| \leq\left\|x_{n}-\bar{x}\right\|+\|\bar{x}-x\| \leq \frac{3}{2} r_{\bar{x}}
$$

Furthermore, using (3.7) and (3.16), one has that, for each $i=0,1$,

$$
\begin{aligned}
\left\|a_{i}^{n}-\bar{y}\right\| & \leq\left\|t_{i}-\bar{y}\right\|+\left\|f\left(x_{n+i-1}\right)-f(x)-\nabla f(x)\left(x_{n+i-1}-x\right)-\frac{1}{2} \nabla^{2} f(x)\left(x_{n+i-1}-x\right)^{2}\right\| \\
& +\left\|f\left(x_{n+i-1}\right)-f(\bar{x})-\nabla f(\bar{x})\left(x_{n+i-1}-\bar{x}\right)-\frac{1}{2} \nabla^{2} f(\bar{x})\left(x_{n+i-1}-\bar{x}\right)^{2}\right\| \\
& \leq \alpha+\frac{L}{(p+1)(p+2)}\left(\left\|x_{n+i-1}-x\right\|^{p+2}+\left\|x_{n+i-1}-\bar{x}\right\|^{p+2}\right) \\
& \leq \alpha+\frac{L}{(p+1)(p+2)}\left(\frac{3^{p+2} r_{\bar{x}}^{p+2}}{2^{p+2}}+r_{\bar{x}}^{p+2}\right) \\
& =\alpha+\frac{L\left(3^{p+2}+2^{p+2}\right) r_{\bar{x}}^{p+2}}{(p+1)(p+2) 2^{p+2}} .
\end{aligned}
$$


It follows, from the definition of $\alpha$ in (3.5), that $a_{i}^{n} \in \mathbb{B}\left(\bar{y}, r_{\bar{y}}\right)$ for each $i=0,1$. Since assumption (3.8) holds for $k=n$, we have

$$
t_{2} \in f(x)+\nabla f(x)\left(x_{n-1}-x\right)+\nabla f(\bar{x})\left(x_{n}-x_{n-1}\right)+\frac{1}{2} \nabla^{2} f(x)\left(x_{n-1}-x\right)^{2}+\frac{1}{2} \nabla^{2} f(\bar{x})\left[\left(x_{n}-\bar{x}\right)^{2}-\left(x_{n-1}-\bar{x}\right)^{2}\right]+\mathscr{F}\left(x_{n}\right),
$$

which can be rewritten as

$$
\begin{aligned}
t_{2} & \left.+f(\bar{x})+\nabla f(\bar{x})\left(x_{n-1}-\bar{x}\right)\right)+\frac{1}{2} \nabla^{2} f(\bar{x})\left(x_{n-1}-\bar{x}\right)^{2} \in f(x)+\nabla f(x)\left(x_{n-1}-x\right)+\nabla f(\bar{x})\left(x_{n}-x_{n-1}\right)+\frac{1}{2} \nabla^{2} f(x)\left(x_{n-1}-x\right)^{2} \\
& +\frac{1}{2} \nabla^{2} f(\bar{x})\left[\left(x_{n}-\bar{x}\right)^{2}-\left(x_{n-1}-\bar{x}\right)^{2}\right]+\mathscr{F}\left(x_{n}\right)+f(\bar{x})+\nabla f(\bar{x})\left(x_{n-1}-\bar{x}\right)+\frac{1}{2} \nabla^{2} f(\bar{x})\left(x_{n-1}-\bar{x}\right)^{2} .
\end{aligned}
$$

Then by the definition of $a_{0}^{n}$, we have that $a_{0}^{n} \in f(\bar{x})+\nabla f(\bar{x})\left(x_{n}-\bar{x}\right)+\frac{1}{2} \nabla^{2} f(\bar{x})\left(x_{n}-\bar{x}\right)^{2}+\mathscr{F}\left(x_{n}\right)$. This, together with (3.2) and (3.15), yields that

$$
x_{n} \in T_{\bar{x}}^{-1}\left(a_{0}^{n}\right) \cap \mathbb{B}\left(\bar{x}, r_{\bar{x}}\right) .
$$

Using (3.4) again, there exists an element $x_{n+1} \in T_{\bar{x}}^{-1}\left(a_{1}^{n}\right)$ such that

$$
\left\|x_{n+1}-x_{n}\right\| \leq M\left\|a_{0}^{n}-a_{1}^{n}\right\| \leq \frac{5 M}{2}\left\|t_{1}-t_{2}\right\|\left(\frac{5 M L r_{\bar{x}}^{p}}{2^{p+1}}\right)^{n-1}
$$

where the last inequality holds by (3.14). By the definition of $a_{1}^{n}$, we have

$$
x_{n+1} \in T_{\bar{x}}^{-1}\left(a_{1}^{n}\right)=T_{\bar{x}}^{-1}\left(t_{2}-f(x)-\nabla f(x)\left(x_{n}-x\right)-\frac{1}{2} \nabla^{2} f(x)\left(x_{n}-x\right)^{2}+f(\bar{x})+\nabla f(\bar{x})\left(x_{n}-\bar{x}\right)+\frac{1}{2} \nabla^{2} f(\bar{x})\left(x_{n}-\bar{x}\right)^{2}\right),
$$

which, together with (3.2), implies that

$$
t_{2} \in f(x)+\nabla f(x)\left(x_{n}-x\right)+\nabla f(\bar{x})\left(x_{n+1}-x_{n}\right)+\frac{1}{2} \nabla^{2} f(x)\left(x_{n}-x\right)^{2}+\frac{1}{2} \nabla^{2} f(\bar{x})\left(\left(x_{n+1}-\bar{x}\right)^{2}-\left(x_{n}-\bar{x}\right)^{2}\right)+\mathscr{F}\left(x_{n+1}\right) .
$$

This, together with (3.17), completes the induction step and ensure the existence of a sequence $\left\{x_{n}\right\}$ satisfying (3.8) and (3.9). Since $\frac{5 M L r_{\bar{x}}^{p}}{2^{p+1}}<1$, we see from (3.9) that $\left\{x_{k}\right\}$ is a Cauchy sequence and hence it is convergent, say to $u^{\prime \prime}$, that is $u^{\prime \prime}:=\lim _{k \rightarrow \infty} x_{k}$. Note that $\mathscr{F}$ has closed graph. Then, taking limit in (3.8), we get $t_{2} \in f(x)+\nabla f(x)\left(u^{\prime \prime}-x\right)+\frac{1}{2} \nabla^{2} f(x)\left(u^{\prime \prime}-x\right)^{2}+\mathscr{F}\left(u^{\prime \prime}\right)$ and so $u^{\prime \prime} \in T_{x}^{-1}\left(t_{2}\right)$. Moreover,

$$
\left\|u^{\prime}-u^{\prime \prime}\right\| \leq \limsup _{n \rightarrow \infty} \sum_{k=2}^{n}\left\|x_{k}-x_{k-1}\right\| \leq \lim _{n \rightarrow \infty} \sum_{k=2}^{n} \frac{5 M}{2}\left\|t_{1}-t_{2}\right\|\left(\frac{5 M L r_{\bar{x}}^{p}}{2^{p+1}}\right)^{k-2}=\frac{5 M 2^{p}}{2^{p+1}-5 M L r_{\bar{x}}^{p}}\left\|t_{1}-t_{2}\right\| .
$$

This completes the proof of the Lemma 3.2.

Before going to demonstrate our main results, we define, for each $x \in X$, the mapping $J_{x}: X \rightarrow Y$ by

$$
J_{x}(\cdot):=f(\bar{x})+\nabla f(\bar{x})(\cdot-\bar{x})+\frac{1}{2} \nabla^{2} f(\bar{x})(\cdot-\bar{x})^{2}-f(x)-\nabla f(x)(\cdot-x)-\frac{1}{2} \nabla^{2} f(x)(\cdot-x)^{2},
$$

and the set-valued mapping $\Phi_{x}: X \rightrightarrows 2^{X}$ by

$$
\Phi_{x}(\cdot)=T_{\bar{x}}^{-1}\left[J_{x}(\cdot)\right] .
$$

Then, for any $x^{\prime}, x^{\prime \prime} \in X$, we have that

$$
\begin{aligned}
\left\|J_{x}\left(x^{\prime}\right)-J_{x}\left(x^{\prime \prime}\right)\right\| & =\left\|(\nabla f(\bar{x})-\nabla f(x))\left(x^{\prime}-x^{\prime \prime}\right)+\frac{1}{2} \nabla^{2} f(\bar{x})\left(\left(x^{\prime}-\bar{x}\right)^{2}-\left(x^{\prime \prime}-\bar{x}\right)^{2}\right)-\frac{1}{2} \nabla^{2} f(x)\left(\left(x^{\prime}-x\right)^{2}-\left(x^{\prime \prime}-x\right)^{2}\right)\right\| \\
& =\left\|(\nabla f(\bar{x})-\nabla f(x))\left(x^{\prime}-x^{\prime \prime}\right)+\frac{1}{2} \nabla^{2} f(\bar{x})\left(\left(x^{\prime}-x^{\prime \prime}+x^{\prime \prime}-\bar{x}\right)^{2}-\left(x^{\prime \prime}-\bar{x}\right)^{2}\right)-\frac{1}{2} \nabla^{2} f(x)\left(\left(x^{\prime}-x^{\prime \prime}+x^{\prime \prime}-x\right)^{2}-\left(x^{\prime \prime}-x\right)^{2}\right)\right\| \\
& \leq\|\nabla f(\bar{x})-\nabla f(x)\|\left\|x^{\prime}-x^{\prime \prime}\right\|+\frac{1}{2}\left\|\nabla^{2} f(\bar{x})-\nabla^{2} f(x)\right\|\left\|x^{\prime}-x^{\prime \prime}\right\|^{2}+\left\|\nabla^{2} f(\bar{x})\left(x^{\prime \prime}-\bar{x}\right)-\nabla^{2} f(x)\left(x^{\prime \prime}-x\right)\right\|\left\|x^{\prime}-x^{\prime \prime}\right\| .
\end{aligned}
$$

\subsection{Superquadratic convergence}

This subsection is devoted to study that if $\nabla^{2} f$ is $(L, p)$-Hölder continuous, the sequence generated by Algorithm 2 converges superquadratically to the solution of (1.1). Thus, the main theorem of this study, which gives some sufficient conditions confirming the convergence of the modified superquadratic method with starting point $x_{0}$, read as follows:

Theorem 3.3. Let $p \in(0,1]$ and $\eta \in(1, \infty)$. Suppose that $T_{\bar{x}}^{-1}$ is Lipschitz-like on $\mathbb{B}\left(\bar{y}, r_{\bar{y}}\right)$ relative to $\mathbb{B}\left(\bar{x}, r_{\bar{x}}\right)$ with constant $M$ and that $\nabla^{2} f$ is $(L, p)$-Höder continuous on $\mathbb{B}\left(\bar{x}, \frac{r_{\bar{x}}}{2}\right)$ with constant $L$. Let $\alpha$ be defined by (3.5) such that (3.6) is hold. Let $\delta>0$ be such that

(a) $\delta \leq \min \left\{\frac{r_{\bar{x}}}{4}, \frac{2(p+1)(p+2) r_{\bar{y}}}{L\left(2^{p+3}+2 \cdot 4^{p+2}+1\right)}, \frac{1285 \alpha}{3 \cdot 2^{p}}, 1\right\}$;

(b) $5(M+1) L\left(\eta 2^{p} \delta^{p+1}+4^{4-p}(p+1)(p+2) r_{\bar{x}}^{p}\right) \leq 2^{p+1}(p+1)(p+2)$; 
(c) $\|\bar{y}\|<\frac{L \delta^{p+2}}{2(p+1)(p+2)}$.

Suppose that $f+\mathscr{F}$ is continuous at $\bar{x}$ for $\bar{y}$, i.e. (3.3) is hold. Then there exists some $\hat{\delta}>0$ such that any sequence $\left\{x_{n}\right\}$ generated by Algorithm 2 with initial point in $\mathbb{B}(\bar{x}, \hat{\delta})$ converges superquadratically to a solution $x^{*}$ of (1.1).

Proof. According to the continuity of $f+\mathscr{F}$ at $\bar{x}$ for $\bar{y}$ and assumption (c), we can choose $0<\hat{\delta} \leq \delta$ be such that

$$
\operatorname{dist}\left(0, f\left(x_{0}\right)+F\left(x_{0}\right)\right) \leq \frac{L \delta^{p+2}}{2(p+1)(p+2)} \quad \text { for each } x_{0} \in \mathbb{B}(\bar{x}, \hat{\boldsymbol{\delta}}) .
$$

Setting

$$
t:=\frac{5 \eta M L 2^{p} \delta^{p+1}}{(p+1)(p+2)\left(2^{p+1}-5 M L r_{\bar{x}}^{p}\right)} .
$$

It follows, from assumption (b), that

$$
5 M L\left(\eta 2^{p} \delta^{p+1}+(p+1)(p+2) r_{\bar{x}}^{p}\right) \leq 5(M+1) L\left(\eta 2^{p} \delta^{p+1}+4^{4-p}(p+1)(p+2) r_{\bar{x}}^{p}\right) \leq 2^{p+1}(p+1)(p+2) .
$$

The above inequality implies that

$$
t \leq 1 \text {. }
$$

Let $x_{0} \in \mathbb{B}(\bar{x}, \hat{\delta})$. We use mathematical induction on $n$ to show that Algorithm 2 generates at least one sequence and every sequence $\left\{x_{n}\right\}$ obtained by Algorithm 2 satisfies the following assertions:

$$
\left\|x_{n}-\bar{x}\right\| \leq 2 \delta
$$

and

$$
\left\|d_{n}\right\| \leq t\left(\frac{1}{2}\right)^{(p+2)^{n}} \delta
$$

for each $n=0,1,2, \ldots$. Now, define

$$
r_{x}:=\frac{9}{(p+1)(p+2)}\left(M L\|x-\bar{x}\|^{p+2}+(p+1)(p+2) M\|\bar{y}\|\right) \quad \text { for each } x \in X .
$$

Because $\eta>1, p \in(0,1]$ and $\delta \leq \frac{r_{\bar{x}}}{4}$ by assumption (a), it follows, from assumption (b), that

$$
\begin{aligned}
257(M+1) L \delta^{p+1} & =(M+1) L\left(\delta^{p+1}+4^{4} \delta^{p+1}\right) \leq(M+1) L\left(\delta^{p+1}+4^{4} \delta^{p}\right) \\
& \leq(M+1) L\left(2^{p} \eta \delta^{p+1}+4^{4-p}(p+1)(p+2) r_{\bar{x}}^{p}\right) \\
& \leq \frac{2^{p+1}(p+1)(p+2)}{5}
\end{aligned}
$$

which gives

$$
M L \delta^{p+1} \leq \frac{2^{p+1}(p+1)(p+2)}{1285} \quad \text { and } \quad L \delta^{p+1} \leq \frac{2^{p+1}(p+1)(p+2)}{1285} .
$$

Thus, by $3 \cdot 2^{p} \delta \leq 1285 \alpha$ in assumption (a) and second inequality in (3.24), we obtain that

$$
\|\bar{y}\|<\frac{L \delta^{p+2}}{2(p+1)(p+2)}=\frac{L \delta^{p+1}}{2(p+1)(p+2)} \cdot \delta \leq \frac{\alpha}{3}
$$

(thanks to assumption (c)). Thus, we obtain from (3.23), together with assumption (c), that

$$
\begin{aligned}
r_{x} & <\frac{9}{2(p+1)(p+2)}\left(2^{p+3} M L \delta^{p+2}+M L \delta^{p+2}\right) \\
& =\frac{9\left(2^{p+3}+1\right) M L}{2(p+1)(p+2)} \delta^{p+2} \\
& =\frac{9\left(2^{p+3}+1\right) M L \delta^{p+1}}{2(p+1)(p+2)} \cdot \delta \quad \text { for each } x \in \mathbb{B}(\bar{x}, 2 \delta) .
\end{aligned}
$$

Since $p \in(0,1]$, by the first inequality in (3.24) we have from (3.26) that

$$
r_{x} \leq 2 \delta \text {. }
$$

It is clear that $\alpha>0$ by assumption (a). Then we have from (3.5) that

$$
\alpha>0 \Rightarrow 2^{p+1}-5 M L r_{\bar{x}}^{p}>0 \Rightarrow 5 M L r_{\bar{x}}^{p}<2^{p+1} .
$$


Therefore, with the help of above relation we obtain that

$$
5 \cdot 4^{p} M L \delta^{p}<5 M L r_{\bar{x}}^{p}<2^{p+1} \Rightarrow L M \delta^{p}<\frac{2^{p+1}}{5 \cdot 4^{p}} .
$$

Note that, for $n=0,(3.21)$ is trivial. To show that the point $x_{1}$ exists and (3.22) holds for $n=0$, it suffices to prove that $\mathscr{D}\left(x_{0}\right) \neq \emptyset$. We will do that by applying Lemma 2.4 to the mapping $\Phi:=\Phi_{x_{0}}$. To do this, let us check that both assumptions (2.1) and (2.2) of Lemma 2.4 hold with $c:=r_{x_{0}}$ and $r:=\frac{8}{9}$. Here, we note that $\bar{x} \in T_{\bar{x}}^{-1}(\bar{y}) \cap \mathbb{B}(\bar{x}, \delta)$. Then by the definition of the excess $e$, we obtain that

$$
\begin{aligned}
\operatorname{dist}\left(\bar{x}, \Phi_{x_{0}}(\bar{x})\right) & \leq e\left(T_{\bar{x}}^{-1}(\bar{y}) \cap \mathbb{B}(\bar{x}, \delta), \Phi_{x_{0}}(\bar{x})\right) \\
& \leq e\left(T_{\bar{x}}^{-1}(\bar{y}) \cap \mathbb{B}\left(\bar{x}, r_{\bar{x}}\right), T_{\bar{x}}^{-1}\left[J_{x_{0}}(\bar{x})\right]\right) .
\end{aligned}
$$

By the $(L, p)$-Hölder continuity property of $\nabla^{2} f$ and (3.14), we obtain, for each $x \in \mathbb{B}(\bar{x}, 2 \delta) \subseteq \mathbb{B}\left(\bar{x}, \frac{r_{\bar{x}}}{2}\right)$, that

$$
\begin{aligned}
\left\|J_{x_{0}}(x)-\bar{y}\right\| & =\left\|f(\bar{x})+\nabla f(\bar{x})(x-\bar{x})+\frac{1}{2} \nabla^{2} f(\bar{x})(x-\bar{x})^{2}-f\left(x_{0}\right)-\nabla f\left(x_{0}\right)\left(x-x_{0}\right)-\frac{1}{2} \nabla^{2} f\left(x_{0}\right)\left(x-x_{0}\right)^{2}-\bar{y}\right\| \\
& \leq\left\|f(x)-f\left(x_{0}\right)-\nabla f\left(x_{0}\right)\left(x-x_{0}\right)-\frac{1}{2} \nabla^{2} f\left(x_{0}\right)\left(x-x_{0}\right)^{2}\right\|+\left\|f(x)-f(\bar{x})-\nabla f(\bar{x})(x-\bar{x})+\frac{1}{2} \nabla^{2} f(\bar{x})(x-\bar{x})^{2}\right\|+\|\bar{y}\| \\
& \leq \frac{L}{(p+1)(p+2)}\left(\left\|x-x_{0}\right\|^{p+2}+\|x-\bar{x}\|^{p+2}\right)+\|\bar{y}\| .
\end{aligned}
$$

Because of $\left\|x_{0}-\bar{x}\right\| \leq \hat{\delta} \leq \delta, L\left(2^{p+3}+2 \cdot 4^{p+2}+1\right) \delta \leq 2(p+1)(p+2) r_{\bar{y}}, \delta \leq 1$ by assumption (a), $\|\bar{y}\|<\frac{L \delta^{p+2}}{2(p+1)(p+2)}$ by assumption (c) and second relation in (3.24), (3.29) implies that

$$
\begin{aligned}
\left\|J_{x_{0}}(x)-\bar{y}\right\| & \leq \frac{L}{(p+1)(p+2)}\left(\left\|(x-\bar{x})+\left(\bar{x}-x_{0}\right)\right\|^{p+2}+\|x-\bar{x}\|^{p+2}\right)+\|\bar{y}\| \\
& \leq \frac{L}{(p+1)(p+2)}\left((3 \delta)^{p+2}+(2 \delta)^{p+2}\right)+\frac{L \delta^{p+2}}{2(p+1)(p+2)} \\
& \leq \frac{L}{2(p+1)(p+2)}\left(2 \cdot 3^{p+2}+2^{p+3}+1\right) \delta^{p+2} \\
& \leq \frac{L}{2(p+1)(p+2)}\left(2 \cdot 3^{p+2}+2^{p+3}+1\right) \delta, \text { since } \delta^{p+1} \leq 1 \\
& \leq r_{\bar{y}} .
\end{aligned}
$$

This means that, for each $x \in \mathbb{B}(\bar{x}, 2 \delta), J_{x_{0}}(x) \in \mathbb{B}\left(\bar{y}, r_{\bar{y}}\right)$. In particular case, putting $x=\bar{x}$ in (3.29). Then we have that

$$
\begin{aligned}
\left\|J_{x_{0}}(\bar{x})-\bar{y}\right\| & \leq \frac{L}{(p+1)(p+2)}\left\|\bar{x}-x_{0}\right\|^{p+2}+\|\bar{y}\| \\
& \leq \frac{3 L}{2(p+1)(p+2)} \delta^{p+2} \leq \frac{3 L}{2(p+1)(p+2)} \delta \\
& \leq r_{\bar{y}} .
\end{aligned}
$$

Hence, by (3.31) and the Lipschitz-like property of $T_{\bar{x}}^{-1}$, we have, from (3.28), that

$$
\begin{aligned}
\operatorname{dist}\left(\bar{x}, \Phi_{x_{0}}(\bar{x})\right) & \leq M\left\|\bar{y}-J_{x_{0}}(\bar{x})\right\| \\
& \leq \frac{1}{(p+1)(p+2)}\left(M L\left\|\bar{x}-x_{0}\right\|^{p+2}+(p+1)(p+2) M\|\bar{y}\|\right) \\
& =\left(1-\frac{8}{9}\right) r_{x_{0}}=c(1-r),
\end{aligned}
$$

which shows that the assumption (2.1) of Lemma 2.4 is satisfied.

Next, we show that assumption (2.2) of Lemma 2.4 is satisfied. To do this, let $x^{\prime}, x^{\prime \prime} \in \mathbb{B}\left(\bar{x}, r_{x_{0}}\right)$. Then we have that $x^{\prime}, x^{\prime \prime} \in \mathbb{B}\left(\bar{x}, r_{x_{0}}\right) \subseteq$ $\mathbb{B}(\bar{x}, 2 \delta) \subseteq \mathbb{B}\left(\bar{x}, r_{\bar{x}}\right)$ by (3.26) and $J_{x_{0}}\left(x^{\prime}\right), J_{x_{0}}\left(x^{\prime \prime}\right) \in \mathbb{B}\left(\bar{y}, r_{\bar{y}}\right)$ by (3.30). This, together with the Lipschitz-like property of $T_{\bar{x}}^{-1}$, implies that

$$
\begin{aligned}
e\left(\Phi_{x_{0}}\left(x^{\prime}\right) \cap \mathbb{B}\left(\bar{x}, r_{x_{0}}\right), \Phi_{x_{0}}\left(x^{\prime \prime}\right)\right) & \leq e\left(\Phi_{x_{0}}\left(x^{\prime}\right) \cap \mathbb{B}\left(\bar{x}, r_{\bar{x}}\right), \Phi_{x_{0}}\left(x^{\prime \prime}\right)\right) \\
& =e\left(T_{\bar{x}}^{-1}\left[J_{x_{0}}\left(x^{\prime}\right)\right] \cap \mathbb{B}\left(\bar{x}, r_{\bar{x}}\right), T_{\bar{x}}^{-1}\left[J_{x_{0}}\left(x^{\prime \prime}\right)\right]\right) \\
& \leq M\left\|J_{x_{0}}\left(x^{\prime}\right)-J_{x_{0}}\left(x^{\prime \prime}\right)\right\| .
\end{aligned}
$$

Since $\nabla^{2} f$ and $\nabla^{2} f(\cdot)(z-\cdot)$ are $(L, p)$-Hölder continuous on $\mathbb{B}\left(\bar{x}, \frac{r_{\bar{x}}}{2}\right)$ for all $z \in \mathbb{B}\left(\bar{x}, \frac{r_{\bar{x}}}{2}\right)$, then $\nabla f$ is also $(L, p)$-Hölder continuous on $\mathbb{B}\left(\bar{x}, \frac{r_{\bar{x}}}{2}\right)$ and for simplicity we take the same constant $L$. Thus, for the choice of $x_{0},(3.18)$ yields that

$$
\begin{aligned}
\left\|J_{x_{0}}\left(x^{\prime}\right)-J_{x_{0}}\left(x^{\prime \prime}\right)\right\| & \leq\left\|\nabla f(\bar{x})-\nabla f\left(x_{0}\right)\right\|\left\|x^{\prime}-x^{\prime \prime}\right\|+\frac{1}{2}\left\|\nabla^{2} f(\bar{x})-\nabla^{2} f\left(x_{0}\right)\right\|\left\|x^{\prime}-x^{\prime \prime}\right\|^{2}+\left\|\nabla^{2} f(\bar{x})\left(x^{\prime \prime}-\bar{x}\right)-\nabla^{2} f\left(x_{0}\right)\left(x^{\prime \prime}-x_{0}\right)\right\|\left\|x^{\prime}-x^{\prime \prime}\right\| \\
& \leq L\left\|\bar{x}-x_{0}\right\|^{p}\left\|x^{\prime}-x^{\prime \prime}\right\|+\frac{L}{2}\left\|\bar{x}-x_{0}\right\|^{p}\left\|x^{\prime}-x^{\prime \prime}\right\|^{2}+L\left\|\bar{x}-x_{0}\right\|^{p}\left\|x^{\prime}-x^{\prime \prime}\right\| \\
& \leq\left(2 L+\frac{L}{2}\left\|x^{\prime}-x^{\prime \prime}\right\|\right)\left\|\bar{x}-x_{0}\right\|^{p}\left\|x^{\prime}-x^{\prime \prime}\right\| \\
& \leq 2 L\left(\delta^{p}+\delta^{p+1}\right)\left\|x^{\prime}-x^{\prime \prime}\right\| .
\end{aligned}
$$


Applying the first inequality of (3.24) and (3.27), it follows, from (3.27), that

$$
\begin{aligned}
e\left(\Phi_{x_{0}}\left(x^{\prime}\right) \cap \mathbb{B}\left(\bar{x}, r_{x_{0}}\right), \Phi_{x_{0}}\left(x^{\prime \prime}\right)\right) \leq 2 M L\left(\delta^{p}+\delta^{p+1}\right)\left\|x^{\prime}-x^{\prime \prime}\right\| & \leq 2^{p+2}\left(\frac{1}{5 \cdot 4^{p}}+\frac{(p+1)(p+2)}{1285}\right)\left\|x^{\prime}-x^{\prime \prime}\right\| \\
& <\frac{8}{9}\left\|x^{\prime}-x^{\prime \prime}\right\|=r\left\|x^{\prime}-x^{\prime \prime}\right\|, \text { since } p \in(0,1] .
\end{aligned}
$$

This means that the assumption (2.2) of Lemma 2.4 is also satisfied. Thus by Lemma 2.4, we can deduce the existence of a fixed point $\hat{x}_{1} \in \mathbb{B}\left(\bar{x}, r_{x_{0}}\right)$ such that $\hat{x}_{1} \in \Phi_{x_{0}}\left(\hat{x}_{1}\right)$, which translates to $0 \in f\left(x_{0}\right)+\nabla f\left(x_{0}\right)\left(\hat{x}_{1}-x_{0}\right)+\frac{1}{2} \nabla^{2} f\left(x_{0}\right)\left(\hat{x}_{1}-x_{0}\right)^{2}+\mathscr{F}\left(\hat{x}_{1}\right)$ and hence $\mathscr{D}\left(x_{0}\right) \neq \emptyset$. Consequently, we can choose $d_{0} \in \mathscr{D}\left(x_{0}\right)$ such that

$$
\left\|d_{0}\right\| \leq \eta \operatorname{dist}\left(0, \mathscr{D}\left(x_{0}\right)\right)
$$

Therefore, according to the Algorithm 2, we can say that $x_{1}:=x_{0}+d_{0}$ is defined.

Now, we will show that the assertion (3.22) is also hold for $n=0$. Note by assumption (a) that $x_{0} \in \mathbb{B}(\bar{x}, \hat{\delta}) \subseteq \mathbb{B}(\bar{x}, \delta) \subseteq \mathbb{B}\left(\bar{x}, \frac{r_{\bar{x}}}{2}\right)$. Since $T_{\bar{x}}^{-1}$ is Lipschitz-like on $\mathbb{B}\left(\bar{y}, r_{\bar{y}}\right)$ relative to $\mathbb{B}\left(\bar{x}, r_{\bar{x}}\right)$, it follows from Lemma 3.2 that $T_{x_{0}}^{-1}$ is Lipschitz-like on $\mathbb{B}(\bar{y}, \alpha)$ relative to $\mathbb{B}\left(\bar{x}, \frac{r_{\bar{x}}}{2}\right)$ with constant $\frac{5 M 2^{p}}{2^{p+1}-5 M L r_{\bar{x}}^{p}}$. Moreover, (3.19) and (3.25) imply that

$$
\operatorname{dist}\left(0, T_{x_{0}}\left(x_{0}\right)=\operatorname{dist}\left(0, f\left(x_{0}\right)+F\left(x_{0}\right)\right) \leq \frac{L \delta^{p+2}}{2(p+1)(p+2)} \leq \frac{\alpha}{3}\right.
$$

It has been mentioned earlier that $x_{0} \in \mathbb{B}\left(\bar{x}, \frac{r_{\bar{x}}}{2}\right)$ and by (3.25)) we have $0 \in \mathbb{B}\left(\bar{y}, \frac{\alpha}{3}\right)$. This, together with (3.33), implies that Lemma 2.3 is applicable and hence by applying it we have that

$$
\operatorname{dist}\left(x_{0}, T_{x_{0}}{ }^{-1}(0)\right) \leq \frac{5 M 2^{p}}{2^{p+1}-5 M L r_{\bar{x}}^{p}} \operatorname{dist}\left(0, T_{x_{0}}\left(x_{0}\right)\right)
$$

Applying (3.34), we have from (3.1) that

$$
\begin{aligned}
\operatorname{dist}\left(0, \mathscr{D}\left(x_{0}\right)\right) & =\operatorname{dist}\left(x_{0}, T_{x_{0}}^{-1}(0)\right) \\
& \leq \frac{5 M 2^{p}}{2^{p+1}-5 M L r_{\bar{x}}^{p}} \operatorname{dist}\left(0, T_{x_{0}}\left(x_{0}\right)\right)
\end{aligned}
$$

Using (3.35), (3.33) and then (3.20) in (3.32), we obtain that

$$
\begin{aligned}
\left\|x_{1}-x_{0}\right\| & =\left\|d_{0}\right\| \leq \eta \operatorname{dist}\left(0, \mathscr{D}\left(x_{0}\right)\right) \\
& \leq \frac{5 \eta M 2^{p}}{2^{p+1}-5 M L r_{\bar{x}}^{p}} \operatorname{dist}\left(0, T_{x_{0}}\left(x_{0}\right)\right) \\
& \leq \frac{5 \eta M L 2^{p} \delta^{p+2}}{2(p+1)(p+2)\left(2^{p+1}-5 M L r_{\bar{x}}^{p}\right)}=t\left(\frac{1}{2}\right) \delta .
\end{aligned}
$$

This shows that (3.22) is hold for $n=0$.

We assume that the points $x_{1}, x_{2}, \ldots, x_{k}$ are generated by Algorithm 2, and (3.21) and (3.22) are true for $n=0,1, \ldots, k-1$. We show that there exists $x_{k+1}$ such that (3.21) and (3.22) are hold for $n=k$. Since, for each $n \leq k-1,(3.21)$ and (3.22) are true and $t \leq 1$ by (3.20), we have the following inequality

$$
\begin{aligned}
\left\|x_{k}-\bar{x}\right\| & \leq \sum_{i=0}^{k-1}\left\|x_{i+1}-x_{i}\right\|+\left\|x_{0}-\bar{x}\right\| \leq \delta \sum_{i=0}^{k-1} t\left(\frac{1}{2}\right)^{(p+2)^{i}}+\delta \\
& \leq \delta \sum_{i=0}^{k-1}\left(\frac{1}{2}\right)^{(p+2)^{i}}+\delta \leq \delta+\delta=2 \delta .
\end{aligned}
$$

This shows that (3.21) holds for $n=k$. Finally, we will show that (3.22) holds for $n=k$. Now if we use the same arguments that we did for the case when $n=0$, we can prove that $\mathscr{D}\left(x_{k}\right) \neq \emptyset$ and so by Algorithm 2 we can choose $d_{k} \in \mathscr{D}\left(0, x_{k}\right)$ such that

$$
\left\|d_{k}\right\| \leq \eta \operatorname{dist}\left(0, \mathscr{D}\left(x_{k}\right)\right)
$$

that is, the point $x_{k+1}$ exists. Moreover, we have that $T_{x_{k}}^{-1}$ is Lipschitz-like on $\mathbb{B}(\bar{y}, \alpha)$ relative to $\mathbb{B}\left(\bar{x}, \frac{r_{\bar{x}}}{2}\right)$ with constant $\frac{5 M 2^{p}}{2^{p+1}-5 M L r_{\bar{x}}^{p}}$. 
Therefore, we have that

$$
\begin{aligned}
\left\|x_{k+1}-x_{k}\right\| & =\left\|d_{k}\right\| \leq \eta \operatorname{dist}\left(0, \mathscr{D}\left(x_{k}\right)\right) \\
& \leq \frac{5 \eta M 2^{p}}{2^{p+1}-5 M L r_{\bar{x}}^{p}} \operatorname{dist}\left(0, T_{x_{k}}\left(x_{k}\right)\right) \\
& =\frac{5 \eta M 2^{p}}{2^{p+1}-5 M L r_{\bar{x}}^{p}} \operatorname{dist}\left(0, f\left(x_{k}\right)+F\left(x_{k}\right)\right) \\
& \leq \frac{5 \eta M 2^{p}}{2^{p+1}-5 M L r_{\bar{x}}^{p}}\left\|f\left(x_{k}\right)-f\left(x_{k-1}\right)-\nabla f\left(x_{k-1}\right)\left(x_{k}-x_{k-1}\right)-\frac{1}{2} \nabla^{2} f\left(x_{k-1}\right)\left(x_{k}-x_{k-1}\right)^{2}\right\| \\
& \leq \frac{5 \eta M L 2^{p}}{(p+1)(p+2)\left(2^{p+1}-5 M L r_{\bar{x}}^{p}\right)}\left\|x_{k}-x_{k-1}\right\|^{p+2} \\
& \leq \frac{5 \eta M L 2^{p} \delta^{p+1}}{(p+1)(p+2)\left(2^{p+1}-5 M L r_{\bar{x}}^{p}\right)}\left(t\left(\frac{1}{2}\right)^{(p+2)^{k-1}}\right)^{p+2} \delta \\
& \leq t\left(\frac{1}{2}\right)^{(p+2)^{k}} \delta .
\end{aligned}
$$

This implies that (3.22) holds for $n=k$ and therefore the proof of the theorem is completed.

In the special case when $\bar{x}$ is a solution of (1.1) (that is $\bar{y}=0$ in Theorem 3.3), then we have the following corollary which gives the super-quadratically local convergence result for the modified superquadratic method.

Corollary 3.4. Let $p \in(0,1]$ and $\eta>1$. Let $\bar{x}$ be the solution of $(1.1)$ and $T_{\bar{x}}^{-1}$ be pseudo-Lipschitz around $(0, \bar{x})$. Suppose that $\nabla^{2} f$ is $(L, p)$-Hölder continuous around $\bar{x}$. Suppose that

$$
\lim _{x \rightarrow \bar{x}} \operatorname{dist}(0, f(x)+\mathscr{F}(x))=0 .
$$

Then there exists some $\hat{\delta}>0$ such that any sequence $\left\{x_{n}\right\}$ generated by Algorithm 2 with initial point in $\mathbb{B}(\bar{x}, \hat{\delta})$ converges superquadratically to a solution $x^{*}$ of (1.1).

Proof. By our assumption, $T_{\bar{x}}^{-1}$ is pseudo-Lipschitz around $(0, \bar{x})$. Then there exist constants $r_{0}, \beta$ and $M$ such that $T_{\bar{x}}^{-1}$ is Lipschitz-like on $\mathbb{B}\left(0, r_{0}\right)$ relative to $\mathbb{B}(\bar{x}, \beta)$ with constant $M$. Then, for each $0<r \leq \beta$, one has that

$$
e\left(T_{\bar{x}}^{-1}\left(y_{1}\right) \cap \mathbb{B}(\bar{x}, r), T_{\bar{x}}^{-1}\left(y_{2}\right) \leq M\left\|y_{1}-y_{2}\right\| \quad \text { for any } y_{1}, y_{2} \in \mathbb{B}\left(0, r_{0}\right)\right.
$$

i.e. $T_{\bar{x}}^{-1}$ is Lipschitz-like on $\mathbb{B}\left(0, r_{0}\right)$ relative to $\mathbb{B}(\bar{x}, r)$ with constant $M$. Let $L \in(0,1)$ and $r_{\bar{x}} \in(0, \beta)$ be such that $\frac{r_{\bar{x}}}{2} \leq r, M L r_{\bar{x}}^{p} \leq \frac{2^{p+1}}{5}$ and $r_{0}-\frac{L\left(3^{p+2}+2^{p+2}\right) r_{\bar{x}}^{p+2}}{(p+1)(p+2) 2^{p+2}}>0$. By the $(L, p)$-Hölder continuous property of $\nabla^{2} f$, for each $x, x^{\prime} \in \mathbb{B}\left(\bar{x}, \frac{r_{\bar{x}}}{2}\right)$, we have that

$$
\left\|\nabla^{2} f(x)-\nabla^{2} f\left(x^{\prime}\right)\right\| \leq L\left\|x-x^{\prime}\right\|^{p} .
$$

Choose $\alpha$ so that

$$
\alpha:=\min \left\{r_{0}-\frac{L\left(3^{p+2}+2^{p+2}\right) r_{\bar{x}}^{p+2}}{(p+1)(p+2) 2^{p+2}}, \frac{r_{\bar{x}}\left(2^{p+1}-5 M L r_{\bar{x}}^{p}\right)}{5 M 2^{p+2}}\right\}>0,
$$

and

$$
\min \left\{\frac{r_{\bar{x}}}{4}, \frac{2(p+1)(p+2) r_{0}}{L\left(2^{p+3}+2 \cdot 4^{p+2}+1\right)}, \frac{1285 \alpha}{3 \cdot 2^{p}}\right\}>0 .
$$

Thus we can choose $0<\delta \leq 1$ such that

$$
\delta \leq \min \left\{\frac{r_{\bar{x}}}{4}, \frac{2(p+1)(p+2) r_{0}}{L\left(2^{p+3}+2 \cdot 4^{p+2}+1\right)}, \frac{1285 \alpha}{3 \cdot 2^{p}}\right\} .
$$

and

$$
5(M+1) L\left(\eta 2^{p} \delta^{p+1}+4^{4-p}(p+1)(p+2) r_{\bar{x}}^{p}\right) \leq 2^{p+1}(p+1)(p+2) .
$$

Now one can easily sees that the assumptions (a)-(c) of Theorem 3.3 are hold. Therefore, to complete the proof of the corollary, we can apply Theorem 3.3 . 


\section{Conclusion}

The semilocal and local convergence results for the modified superquadratic method are established with $\eta>1$ under the assumptions that $T_{\bar{x}}^{-1}$ is Lipschitz-like as well as $\nabla^{2} f$ is $(L, p)$-Hölder continuous. This result extends and improves the corresponding one [13]. This result seems new for the generalized equation problem (1.1). According to the main result of this study, we have the following conclusions:

- If $p=0$, then the Fréchet derivative of $f$ satisfies the continuity condition with constant $\mathrm{L}$ and we obtain the quadratic convergence of the modified superquadratic method. In this case the result established in the present paper coincides with the result presented in [22, Theorem 3.1, Corollary 3.1].

- If $p=1$, then the Fréchet derivative of $f$ satisfies the Lipschitz condition and we obtain the cubic convergence of the modified superquadratic method. In this case the result established in the present paper coincides with the result presented in [22, Theorem 3.2, Corrolary 3.2].

\section{Acknowledgments}

This work is fully supported by Ministry of Science and Technology, Bangladesh, Grant No. 39.009.002.01.00.057.2015-2016/EAS-326.

\section{References}

[1] A. L. Dontchev, Local convergence of the Newton method for generalized equation, C. R. A. S Paris Ser.I 322 (1996), 327-331.

[2] A. L. Dontchev, Uniform convergence of the Newton method for Aubin continuous maps, Serdica Math. J. 22 (1996), $385-398$.

[3] A. L. Dontchev, Local analysis of a Newton-type method based on partial linearization, Lectures in Applied Mathematics 32 (1996), 295-306.

[4] A. L. Dontchev and W. W. Hager, An inverse mapping theorem for set-valued maps, Proc. Amer. Math. Soc. 121 (1994), $481-489$.

[5] A. Piétrus, Generalized equations under mild differentiability conditions, Rev. R. Acad. Cienc. Exact. Fis. Nat. 94(1) (2000), 15-18.

[6] A. Piétrus, Does Newton's method for set-valued maps converges uniformly in mild differentiability context?, Rev. Colombiana Mat. 34 (2000), 49-56.

[7] C. Li, W. H. Zhang and X. Q. Jin, Convergence and uniqueness properties of Gauss-Newton's method, Comput. Math. Appl. 47 (2004), $1057-1067$.

[8] J. P. Dedieu and M. H. Kim, Newton's method for analytic systems of equations with constant rank derivatives, J. Complexity 18 (2002), $187-209$.

[9] J. P. Aubin, Lipschitz behavior of solutions to convex minimization problems, Math. Oper. Res. 9 (1984), 87-111.

[10] J. P. Aubin and H. Frankowska, Set-valued Analysis, Birkhäuser, Boston, 1990.

[11] J. P. Dedieu and M. Shub, Newton's method for overdetermined systems of equations, Math. Comp. 69 (2000), 1099-1115.

[12] J. S. He, J. H. Wang and C. Li, Newton's method for underdetemined systems of equations under the modified $\gamma$-condition, Numer. Funct. Anal. Optim. 28 (2007), 663-679.

[13] M. Geoffroy and A. Piétrus, A superquadratic method for solving generalized equations in the Hóder case, Ricerche di Matematica LII (2003), 231-240.

[14] M. Geoffroy, S. Hilout and A. Piétrus, Acceleration of convergence in Dontchev's iterative method for solving variational inclusions, Serdica Math. J. 29 (2003), 45-54

[15] M. Geoffroy, S. Hilout and A. Piétrus, Stability of a cubically convergent method for generalized equations, Set-Valued Analysis 14 (2006), $41-54$.

[16] M. H. Rashid, A Convergence Analysis of Gauss-Newton-type Method for Holder Continuous Maps, Indian Journal of Mathematics 57(2) (2014), $181-198$.

[17] M.H. Rashid, Convergence Analysis of a Variant of Newton-type Method for Generalized Equations, International Journal of Computer Mathematics 95(3) (2018), 584-600.

[18] M.H. Rashid, On the convergence of extended Newton-type method for solving variational inclusions, Cogent Mathematics, 1(1) (2014), DOI 10.1080/23311835.2014.980600.

[19] M. H. Rashid, Convergence Analysis of Extended Hummel-Seebeck-type Method for Solving Variational Inclusions, Vietnam Journal of Mathematics 44 (2016), 709-726.

[20] M.H. Rashid, Extended Newton-type Method and its Convergence Analysis for Nonsmooth Generalized Equations, J. Fixed Point Theory and Appl. 19 (2017), 1295-1313.

[21] M. H. Rashid, J. H. Wang and C. Li, Convergence analysis of a method for variational inclusions, Applicable Analysis 91(10) (2012), $1943-1956$.

[22] M. H. Rashid, M.Z. Ali and A. Pietrus, Extended Cubic Method and Its Convergence Analysis for Generalized Equations, Journal of Advances and Applied Mathematics, 3(3) (2018), 91-108.

[23] M. H. Rashid, S. H. Yu, C. Li and S, Y. Wu, Convergence analysis of the Gauss-Newton-type method for Lipschitz-like mappings, J. Optim. Theory Appl. 158(1) (2013), 216-233.

[24] S. M. Robinson, Generalized equations and their solutions, part I: basic theory, Math. Progamming Stud.10 (1979), $128-141$.

[25] S. M. Robinson, Strong regular generalized equations, Math. of Oper. Res. 5 (1980), 43-62.

[26] S. M. Robinson, Generalized equations and their solutions, part II: applications to nonlinear programming, Math. Programming Stud.19 (1982), $200-221$.

[27] X. B. Xu and C. Li, Convergence of Newton's method for systems of equations with constant rank derivatives, J. Comput. Math. 25 (2007), 705-718.

[28] X. B. Xu and C. Li, Convergence criterion of Newton's method for singular systems with constant rank derivatives, J. Math. Anal. Appl. 345 (2008), 689-701. 